\title{
ОПРЕДЕЛЕНИЕ ЗАВИСИМОСТИ СИЛЫ ГРАВИТАЦИОННОГО ВЗАИМОДЕЙСТВИЯ ТЕЛ ОТ СКОРОСТИ ИХ ДВИЖЕНИЯ
}

\author{
(C) 2021 г. A.A. Грибков* \\ Московский Государственный Технологический Университет «СТАНКИН», Москва \\ В статье представлены результаты проверки гипотезы о зависимости гра- \\ витации от скорости движения тел. Проведенное численное моделирование од- \\ нозначно подтвердило соответствие расчетных и наблюдаемых значений пре- \\ цессии перицетров орбит в двойных системах, включающих пульсары, а так- \\ же планет солнечной системы. На основе выявленной различной зависимости \\ силы гравитации от скорости «источника» $и$ «приемника» гравитачии в ста- \\ тье описывается новый эффект прецессии центров двойных систем, который \\ должен быть найден в результате дальнейших практических исследований.
}

Ключевые слова: гравитация, двойные системы, пульсары

\section{I. ВВЕДЕНИЕ}

Предположение о том, что сила гравитационного взаимодействия тел зависит от скорости их движения, не новое. Теории Ритца, Гербера и др. предполагали наличие такого влияния. Специальная теория относительности Эйнштейна также предполагает влияние скорости движения тел на силу их гравитационного взаимодействия (через изменение масс). В рамках данной статьи предполагается проверить гипотезу, согласно которой сила гравитационного притяжения зависит от скоростей взаимодействующих

*Электронный адрес: andarmo@yandex.ru 
тел следующим образом:

$$
\begin{aligned}
& F_{1 \leftarrow 2}=\frac{G M_{1} M_{2}}{r^{2}}\left(1+\frac{v_{2}^{2}}{w^{2}}\right)^{\mu / 2}\left(1+\frac{v_{1}^{2}}{w^{2}}\right)^{\lambda / 2} \approx \frac{G M_{1} M_{2}}{r^{2}}\left(1+\frac{\mu v_{2}^{2}+\lambda v_{1}^{2}}{2 w^{2}}\right), \\
& F_{2 \leftarrow 1}=\frac{G M_{1} M_{2}}{r^{2}}\left(1+\frac{v_{1}^{2}}{w^{2}}\right)^{\mu / 2}\left(1+\frac{v_{2}^{2}}{w^{2}}\right)^{\lambda / 2} \approx \frac{G M_{1} M_{2}}{r^{2}}\left(1+\frac{\mu v_{1}^{2}+\lambda v_{2}^{2}}{2 w^{2}}\right),
\end{aligned}
$$

где $F_{1 \leftarrow 2}, F_{2 \leftarrow 1}-$ силы, действующие, соответственно, со стороны 2-го тела (с массой $M_{2}$ ) на 1-ое (с массой $M_{1}$ ) и со стороны 1-го тела - на 2-ое, $v_{1}, v_{2}$ - абсолютные значения скоростей 1-го и 2-го тел, $r$ - текущее расстояние между телами, $\mu$ - показатель степени, с которым скорость движения источника гравитации определяет силу гравитации, $\lambda$ - показатель степени, с которым скорость движения «приемника» гравитации (объекта, на который действует гравитация) определяет силу гравитации, $w$ - константа, имеющая размерность скорости $\left(w \gg v_{1}, w \gg v_{2}\right)$.

Ключевым индикатором соответствия предлагаемых гипотез относительно гравитации действительности последние сто лет является возможность на их основе объяснить прецессию перицентров компонентов двойных систем (изначально речь шла о прецессии перигелия Меркурия и других планет солнечной системы). Наиболее выражено данный эффект проявляется в двойных нейтронных звездах (хотя бы одна из нейтронных звезд - пульсар), двойных системах из нейтронной звезды (пульсара) и белого карлика, при определенных условиях - в двойных системах из нейтронной звезды (пульсара) и звезды главной последовательности. Кроме того, значительный интерес представляет исследование данного эффекта в солнечной системе.

\section{II. ЧИСЛЕННОЕ МОДЕЛИРОВАНИЕ ОРБИТАЛЬНОГО ДВИЖЕНИЯ}

\section{А. Алгоритм численного моделирования}

Для различных вариантов зависимости силы гравитации от скорости (т.е. различных $\mu, \lambda$ и $w$ ) было выполнено численное пошаговое моделирование движения компонентов двойных систем. Изложим кратко последовательность расчетов.

Интервал времени, соответствующий каждому шагу:

$$
\tau=\frac{P_{b}}{N},
$$

где $P_{b}$ - орбитальный период двойной системы, $N$ - число шагов на период. 
Определим действующие силы гравитации:

$$
\begin{aligned}
& \left|F_{1 \leftarrow 2 \mid i}\right|=\frac{k}{r_{i-1}^{2}}\left(1+\frac{v_{2 \mid i-1}^{2}}{w^{2}}\right)^{\mu / 2}\left(1+\frac{v_{1 \mid i-1}^{2}}{w^{2}}\right)^{\lambda / 2}, \\
& \left|F_{2 \leftarrow 1 \mid i}\right|=\frac{k}{r_{i-1}^{2}}\left(1+\frac{v_{1 \mid i-1}^{2}}{w^{2}}\right)^{\mu / 2}\left(1+\frac{v_{2 \mid i-1}^{2}}{w^{2}}\right)^{\lambda / 2},
\end{aligned}
$$

где $k=G M_{1} M_{2}$ - постоянный коэффициент, определяемый гравитационной постоянной $G$ и массами компонентов $M_{1}$ и $M_{2} ; r_{i-1}=\sqrt{\left(x_{1 \mid i-1}-x_{2 \mid i-1}\right)^{2}+\left(y_{1 \mid i-1}-y_{2 \mid i-1}\right)^{2}}$ - расстояние между взаимодействующими компонентами на предыдущем шаге, $x_{1 \mid i-1}, x_{2 \mid i-1}, y_{1 \mid i-1}, y_{2 \mid i-1}$ - координаты компонентов на предыдущем шаге.

Разложим силы по осям:

$$
\begin{aligned}
& F_{1 \leftarrow 2 \mid i X}=\frac{x_{2 \mid i-1}-x_{1 \mid i-1}}{r_{i-1}}\left|F_{1 \leftarrow 2 \mid i}\right| ; F_{1 \leftarrow 2 \mid i Y}=\frac{y_{2 \mid i-1}-y_{1 \mid i-1}}{r_{i-1}}\left|F_{1 \leftarrow 2 \mid i}\right| ; \\
& F_{2 \leftarrow 1 \mid i X}=\frac{x_{2 \mid i-1}-x_{1 \mid i-1}}{r_{i-1}}\left|F_{2 \leftarrow 1 \mid i}\right| ; F_{1 \leftarrow 2 \mid i Y}=\frac{y_{2 \mid i-1}-y_{1 \mid i-1}}{r_{i-1}}\left|F_{2 \leftarrow 1 \mid i}\right| ;
\end{aligned}
$$

Определим скорости компонентов:

$$
\begin{aligned}
& v_{1 \mid i X}=v_{1 \mid(i-1) X}+\tau \cdot \frac{F_{1 \leftarrow 2 \mid i X}}{M_{1}} ; v_{1 \mid i Y}=v_{1 \mid(i-1) Y}+\tau \cdot \frac{F_{1 \leftarrow 2 \mid i Y}}{M_{1}} ; \\
& v_{2 \mid i X}=v_{2 \mid(i-1) X}+\tau \cdot \frac{F_{2 \leftarrow 1 \mid i X}}{M_{2}} ; v_{2 \mid i Y}=v_{2 \mid(i-1) Y}+\tau \cdot \frac{F_{2 \leftarrow 1 \mid i Y}}{M_{2}} \\
& v_{1 \mid i}=\sqrt{v_{1 \mid i X}^{2}+v_{1 \mid i Y}^{2}} ; v_{2 \mid i}=\sqrt{v_{2 \mid i X}^{2}+v_{2 \mid i Y}^{2}} .
\end{aligned}
$$

Координаты компонентов:

$$
\begin{aligned}
& x_{1 \mid i}=x_{1 \mid i-1}+\frac{\tau}{2} \cdot\left(v_{1 \mid(i-1) X}+v_{1 \mid i X}\right) ; y_{1 \mid i}=y_{1 \mid i-1}+\frac{\tau}{2} \cdot\left(v_{1 \mid(i-1) Y}+v_{1 \mid i Y}\right) ; \\
& x_{2 \mid i}=x_{2 \mid i-1}+\frac{\tau}{2} \cdot\left(v_{2 \mid(i-1) X}+v_{2 \mid i X}\right) ; y_{2 \mid i}=y_{2 \mid i-1}+\frac{\tau}{2} \cdot\left(v_{2 \mid(i-1) Y}+v_{2 \mid i Y}\right) .
\end{aligned}
$$

Начальные положения и скорости компонентов определяются исходя из имеющихся данных по массам компонентов, орбитальному периоду и эксцентриситету орбиты на момент нахождения компонентов в апоцентрах орбит.

Моделирование проводилось с числом шагов за один период системы $N=1.0 \times 10^{6}$ на $n=10$ периодах. В процессе моделирования определялись положения, в которых расстояние между компонентами системы достигало максимального значение (положения апоцентров; использовались вместо положений перицентров поскольку соответствуют меньшей скорости движения и соответственно более точно определяются). Для таких 
положений проводилось уточнение с минимальным шагом (до $1 / 2^{10}$ от номинального) и находился угол $\varphi_{j}$ между линией апсид и осью $x$ :

$$
\varphi_{j}=\arcsin \left[\left(y_{1(j)}-y_{2(j)}\right) / r_{j}\right]
$$

где $r_{j}$ - текущее значение максимума расстояния между компонентами системы; $y_{1(j)}, y_{2(j)}$ - значения координат $y$ компонентов, соответствующие $r_{j}$.

Значение прецессии перицентра (или апоцентра или линии апсид) определялось следующим образом:

$$
\dot{\omega}=\frac{180}{\pi} \frac{P_{\oplus}}{n P_{b}} \sum_{j=1}^{n}\left(\varphi_{j}-\varphi_{j-1}\right), \text { \% год, }
$$

где $P_{\oplus}=3.156 \times 10^{7}$ с - продолжительность года.

\section{В. Результаты численного моделирования}

Проведенное численное моделирование различных вариантов зависимости силы гравитации от скорости движения источника и «приемника» гравитации (двойных нейтронных звезд, систем нейтронная звезда + белый карлик, системы нейтронная звезда + желтый карлик, а также планет солнечной системы) показали, что соответствие результатов моделирования существующим данным реальных наблюдений обеспечивается при $\mu=3 \kappa, \lambda=1 \kappa(\kappa-$ постоянный множитель):

$$
\begin{aligned}
& F_{1 \leftarrow 2}=\frac{G M_{1} M_{2}}{r^{2}}\left(1+\frac{v_{2}^{2}}{w^{2}}\right)^{3 \kappa / 2}\left(1+\frac{v_{1}^{2}}{w^{2}}\right)^{\kappa / 2} \approx \frac{G M_{1} M_{2}}{r^{2}}\left(1+\kappa \frac{3 v_{2}^{2}+v_{1}^{2}}{2 w^{2}}\right), \\
& F_{2 \leftarrow 1}=\frac{G M_{1} M_{2}}{r^{2}}\left(1+\frac{v_{1}^{2}}{w^{2}}\right)^{3 \kappa / 2}\left(1+\frac{v_{2}^{2}}{w^{2}}\right)^{\kappa / 2} \approx \frac{G M_{1} M_{2}}{r^{2}}\left(1+\kappa \frac{3 v_{1}^{2}+v_{2}^{2}}{2 w^{2}}\right) .
\end{aligned}
$$

При $\kappa=1$ значение $w=1.2247 \times 10^{8} \mathrm{~m} / \mathrm{c}(=c / \sqrt{6}$, где $c-$ скорость света). Результаты численного моделирования движения компонентов двойных нейтронных звезд приведены в табл. I-II. Как можно видеть, расчетные данные с достаточно высокой точностью соответствуют наблюдаемым (или предполагаемым в настоящее время).

Наряду с двойными нейтронными звездами, в качестве объекта для изучения тонких гравитационных эффектов можно использовать системы из нейтронной звезды и белого карлика. В результате рассмотрения четырех таких систем (по которым имеются 
Таблица I. Прецессия перицентров двойных нейтронных звезд

\begin{tabular}{|c|c|c|c|c|c|c|c|}
\hline \multicolumn{2}{|c|}{ Параметр } & \multicolumn{6}{|c|}{ Двойные нейтронные звезды (DNS) } \\
\hline & PSR & $\mathrm{B} 1913+16$ & J0737-3039 & $\mathrm{B} 1534+12$ & $\mathrm{~B} 2127+11 \mathrm{C}$ & $\mathrm{J} 1518+4904$ & J1811-1736 \\
\hline \multicolumn{8}{|c|}{ Исходные данные } \\
\hline \multicolumn{2}{|c|}{$\begin{array}{l}\text { Источник } \\
\text { данных }\end{array}$} & {$[1]$} & {$[2]$} & {$[3]$} & {$[4]$} & {$[5]$} & [6]-[8] \\
\hline \multicolumn{2}{|c|}{$\begin{array}{l}\text { Орбитальный } \\
\text { период, } P_{b}, \text { c }\end{array}$} & 27907 & 8835 & 36352 & 28968 & $7.46 \times 10^{5}$ & $1.63 \times 10^{6}$ \\
\hline \multicolumn{2}{|c|}{$\begin{array}{l}\text { Эксцентри- } \\
\text { ситет, } e\end{array}$} & 0.617 & 0.088 & 0.274 & 0.681 & 0.249 & 0.828 \\
\hline \multicolumn{2}{|c|}{$\begin{array}{l}\text { Прецессия } \\
\text { перицентра, } \\
\dot{\omega}, \% \text { год }\end{array}$} & 4.227 & $16.88(10)$ & 1.756 & 4.464 & 0.01137 & $0.009(2)$ \\
\hline \multirow{2}{*}{$\begin{array}{l}\text { Массы, } \\
M_{\odot}\end{array}$} & $M_{1}$ & 1.441 & $1.337(1)$ & $1.333(1)$ & $1.358(10)$ & $1.05_{-0.14}^{+1.21}$ & $1.17 \ldots 1.6$ \\
\hline & $M_{2}$ & 1.387 & $1.250(1)$ & $1.345(1)$ & $1.354(10)$ & $1.56_{-1.20}^{+0.20}$ & $0.93 \ldots 1.5$ \\
\hline \multicolumn{8}{|c|}{ Выбор наиболее вероятных значений масс } \\
\hline \multirow{2}{*}{$\begin{array}{l}\text { Массы, } \\
M_{\odot}\end{array}$} & $M_{1}$ & 1.441 & 1.337 & 1.333 & 1.358 & 1.05 & $1.35^{* *}$ \\
\hline & $M_{2}$ & 1.387 & 1.250 & 1.345 & 1.354 & $1.67^{*}$ & $1.22^{* * *}$ \\
\hline \multicolumn{8}{|c|}{ Результаты моделирования при $\mu=3, \lambda=1, w=1.2247 \times 10^{8}$ м $/ \mathrm{c}$} \\
\hline$\dot{\omega}$, огод & & $4.225(4)[\sigma]$ & $16.878(7)[\sigma]$ & $1.754(1)[\sigma]$ & $4.461(5)[\sigma]$ & $0.01134(12)[\sigma]$ & $0.00901(5)[\sigma]$ \\
\hline \multicolumn{8}{|c|}{$\begin{array}{l}* \text { - суммарная масса системы } M_{1}+M_{2}=2.7183(7) M_{\odot} \\
\text { ** - среднее наблюдаемое значение массы пульсара } \\
\text { ***_ }\end{array}$} \\
\hline
\end{tabular}

наиболее полные и достоверные данные) получено подтверждение наличия предполагаемой зависимости гравитации от скорости движения (см. табл. III).

В ходе проведенного исследования было осуществлено моделирование движений компонентов двойной системы из пульсара J1903+0327 и желтого карлика (звезды главной последовательности класса G с массой порядка массы Солнца). Желтые карлики - относительно стабильные звезды, для которых нехарактерны многие эффекты, способные повлиять на смещение линии апсид (например, интенсивная потеря массы, свойственная крупным звездам, особенно в двойных системах). Проведенное моделирования подтвердило справедливость предположения о зависимости гравитации от скорости движения.

Важную роль в подтверждении рассматриваемой зависимости гравитации от скоростей источника и «приемника» играет анализ прецессии перигелиев орбит планет солнечной системы: Меркурия, Венеры, Земли и Марса (данные по другим планетам 
Таблица II. Прецессия перицентров двойных нейтронных звезд (продолжение)

\begin{tabular}{|c|c|c|c|c|c|c|}
\hline \multicolumn{2}{|c|}{ Параметр } & \multicolumn{5}{|c|}{ Двойные нейтронные звезды (DNS) } \\
\hline & PSR & $\mathrm{J} 0453+1559$ & J1756-2251 & $\mathrm{J} 1906+0746^{*}$ & $\mathrm{~J} 1829+2456$ & J1930-1852 \\
\hline \multicolumn{7}{|c|}{ Исходные данные } \\
\hline \multicolumn{2}{|c|}{$\begin{array}{l}\text { Источник } \\
\text { данных }\end{array}$} & {$[9]$} & {$[10]$} & {$[11]$} & {$[12],[13]$} & {$[14]$} \\
\hline \multicolumn{2}{|c|}{$\begin{array}{l}\text { Орбитальный } \\
\text { период, } P_{b}, \text { с }\end{array}$} & $3.519 \times 10^{5}$ & $2.762 \times 10^{4}$ & $1.434 \times 10^{4}$ & $1.016 \times 10^{5}$ & $3.893 \times 10^{6}$ \\
\hline \multicolumn{2}{|c|}{$\begin{array}{l}\text { Эксцентри- } \\
\text { ситет, } e\end{array}$} & 0.1125 & 0.1806 & 0.085 & 0.1391 & 0.3989 \\
\hline \multicolumn{2}{|c|}{$\begin{array}{l}\text { Прецессия } \\
\text { перицентра, } \\
\dot{\omega}, \text { \%год }\end{array}$} & 0.0379 & 2.582 & 7.584 & $0.2919(16)$ & $0.00078(4)$ \\
\hline \multirow{2}{*}{$\begin{array}{l}\text { Массы, } \\
M_{\odot} \\
\end{array}$} & $M_{1}$ & $1.559 \pm 0.005$ & $1.341(7)$ & $1.291 \pm 0.011$ & $<1.38^{* *}$ & $<1.32^{* * *}$ \\
\hline & $M_{2}$ & $1.174 \pm 0.004$ & $1.230(7)$ & $1.322 \pm 0.011$ & $>1.22^{* *}$ & $>1.3^{* * *}$ \\
\hline \multicolumn{7}{|c|}{ Выбор наиболее вероятных значений масс } \\
\hline \multirow{2}{*}{$\begin{array}{l}\text { Массы, } \\
M_{\odot} \\
\end{array}$} & $M_{1}$ & 1.559 & 1.341 & 1.291 & $1.3 \pm 0.08$ & $1.2 \pm 0.1$ \\
\hline & $M_{2}$ & 1.174 & 1.230 & 1.322 & $1.3 \mp 0.08$ & $1.4 \mp 0.1$ \\
\hline \multicolumn{7}{|c|}{ Результаты моделирования при $\mu=3, \lambda=1, w=1.2247 \times 10^{8} \mathrm{~m} / \mathrm{c}$} \\
\hline$\dot{\dot{\omega}}$, \%год & & $0.0379(4)[\sigma]$ & $2.580(9)[\sigma]$ & $7.576(12)[\sigma]$ & $0.2926(11)[\sigma]$ & $0.000782(15)[\sigma]$ \\
\hline \multicolumn{7}{|c|}{ * - есть сомнения, что компаньоном пульсара является нейтронная звезда, а не белый карлик [11] } \\
\hline \multicolumn{7}{|c|}{ ** - суммарная масса системы $M_{1}+M_{2}=2.5(2) M_{\odot}$} \\
\hline
\end{tabular}

крайне неточны), а также астероидов (данные имеются только по Икару). Важность анализа орбит планет обусловлена огромным различием масс рассматриваемых космических тел и Солнца, вследствие которого взаимодействие происходит «односторонне»: Солнце воздействует на эти тела, а их влияние на Солнце пренебрежимо мало (в число рассматриваемых планет не включены Юпитер и Сатурн). Результаты численного моделирования согласуются с результатами наблюдений (см. табл. IV).

\section{С. Определение эмпирической зависимости}

Для определения эмпирической формулы (воспроизводимой предлагаемой математической моделью) зависимости расчетной величины прецессии перицентра от входных параметров (масс компонентов $M_{1}$ и $M_{2}$, орбитального периода системы $P_{b}$, эксцентриситета орбит $e$, константы $w$, гравитационной постоянной $G$ ) при $\mu=3, \lambda=1$ было 
Таблица III. Прецессия перицентров двойных систем

[нейтронная звезда + белый карлик] $(\mathrm{NS}+\mathrm{WD})$ и системы

[нейтронная звезда + желтый карлик] $(\mathrm{NS}+\mathrm{GDS})$

\begin{tabular}{|c|c|c|c|c|c|c|}
\hline \multicolumn{2}{|c|}{ Параметр } & \multicolumn{4}{|c|}{$\mathrm{NS}+\mathrm{WD}$} & \multirow{2}{*}{$\begin{array}{c}\mathrm{NS}+\mathrm{GDS} \\
\mathrm{J} 1903+0327\end{array}$} \\
\hline & PSR & J1141-6545 & $\mathrm{B} 2303+46$ & B1802-07 & $\mathrm{J} 0621+1002$ & \\
\hline \multicolumn{7}{|c|}{ Исходные данные } \\
\hline \multicolumn{2}{|c|}{$\begin{array}{l}\text { Источник } \\
\text { данных }\end{array}$} & {$[15]-[17]$} & {$[18]$} & {$[19]$} & {$[20]$} & {$[21],[22]$} \\
\hline \multicolumn{2}{|c|}{$\begin{array}{l}\text { Орбитальный } \\
\text { период, } P_{b}, \text { с }\end{array}$} & $1.708 \times 10^{4}$ & $1.066 \times 10^{6}$ & $2.26 \times 10^{5}$ & $7.19 \times 10^{5}$ & $8.22 \times 10^{6}$ \\
\hline \multicolumn{2}{|c|}{$\begin{array}{l}\text { Эксцентри- } \\
\text { ситет, } e\end{array}$} & 0.1719 & 0.658 & 0.212 & 0.00246 & 0.437 \\
\hline \multicolumn{2}{|c|}{$\begin{array}{l}\text { Прецессия } \\
\text { перицентра, } \\
\dot{\omega}, \text { \%год } \\
\end{array}$} & $5.3084(9)$ & $0.0099(2)$ & $0.058(2)$ & $0.0116(8)$ & $2.400(2) \times 10^{-4}$ \\
\hline \multirow{2}{*}{$\begin{array}{l}\text { Массы, } \\
M_{\odot} \\
\end{array}$} & $M_{1}$ & $1.27(1)$ & $1.16(28)$ & $1.26_{-0.67}^{+0.15}$ & $1.70_{-0.29}^{+0.32}$ & $1.667(21)$ \\
\hline & $M_{2}$ & $1.02(1)$ & $1.37(24)$ & $0.36_{-0.22}^{+0.56}$ & $0.97_{-0.15}^{+0.27}$ & $1.029(8)$ \\
\hline \multicolumn{7}{|c|}{ Выбор наиболее вероятных значений масс } \\
\hline \multirow{2}{*}{$\begin{array}{l}\text { Массы, } \\
M_{\odot}\end{array}$} & $M_{1}$ & 1.27 & 1.16 & 1.26 & 1.70 & 1.667 \\
\hline & $M_{2}$ & 1.02 & 1.37 & 0.36 & 0.97 & 1.029 \\
\hline \multicolumn{7}{|c|}{ Результаты моделирования при $\mu=3, \lambda=1, w=1.2247 \times 10^{8} \mathrm{~m} / \mathrm{c}$} \\
\hline$\dot{\dot{\omega}, ~ \% г о д ~}$ & & $5.306(10)[\sigma]$ & $0.00992(2)[\sigma]$ & $0.0578(3)[\sigma]$ & $0.010(1)[\sigma]$ & $2.40(4) \times 10^{-4}[\sigma]$ \\
\hline
\end{tabular}

Таблица IV. Аномальная прецессия перигелиев орбит в Солнечной системе

\begin{tabular}{|c|c|c|c|c|c|c|}
\hline \multirow{2}{*}{\multicolumn{2}{|c|}{ Параметр }} & \multicolumn{4}{|c|}{ Планеты } & \multirow{2}{*}{$\frac{\text { Астероид }}{\text { Икар }}$} \\
\hline & & Меркурий & Венера & Земля & Mapc & \\
\hline \multicolumn{7}{|c|}{ Исходные данные [23] } \\
\hline \multicolumn{2}{|c|}{$\begin{array}{l}\text { Орбитальный } \\
\text { период, } P_{b}, \text { с }\end{array}$} & $7.6005 \times 10^{6}$ & $1.9414 \times 10^{7}$ & $3.1558 \times 10^{7}$ & $5.9329 \times 10^{7}$ & $3.5320 \times 10^{7}$ \\
\hline \multicolumn{2}{|l|}{$\begin{array}{l}\text { Эксцентри- } \\
\text { ситет, } e\end{array}$} & 0.2056 & 0.0068 & 0.0167 & 0.0934 & 0.82684 \\
\hline \multicolumn{2}{|c|}{$\begin{array}{l}\text { Прецессия } \\
\text { перицентра, } \\
\dot{\omega},{ }^{\prime \prime} / 100 \text { лет }\end{array}$} & $43.1 \pm 0.5$ & $8.4 \pm 4.8$ & $5.0 \pm 1.2$ & $1.1 \pm 0.3$ & $9.8 \pm 0.8$ \\
\hline \multirow{2}{*}{ Массы, кг } & $M_{p}$ & $3.2868 \times 10^{23}$ & $4.8107 \times 10^{24}$ & $5.976 \times 10^{24}$ & $6.3345 \times 10^{23}$ & $2.9 \times 10^{12}$ \\
\hline & $M_{\odot}$ & \multicolumn{5}{|c|}{$1.989 \times 10^{30}$} \\
\hline \multicolumn{7}{|c|}{ Результаты моделирования* при $\mu=3, \lambda=1, w=1.2247 \times 10^{8}$ м $/ \mathrm{c}$} \\
\hline$\overline{\dot{\omega}, ~}{ }^{\prime \prime} / 100$ ле & & $43.01(1)[\sigma]$ & $8.623(2)[\sigma]$ & $3.837(1)[\sigma]$ & $1.3510(1)[\sigma]$ & $10.13(5)[\sigma]$ \\
\hline
\end{tabular}


выполнено варьирование значениями параметров (по отдельности $M_{1}, M_{2}, P_{b}, e, w$ и $G$ при постоянных прочих параметрах). При варьировании $M_{1}$ и $M_{2}$ исследовалось изменение прецессии в зависимости от функции $f_{1}=\left(M_{1} M_{2}\right)^{\alpha} \times\left(M_{1}+M_{2}\right)^{\beta}$, при варьировании $e-$ изменение прецессии в зависимости от функции $f_{2}=e^{\xi} \times\left(1-e^{2}\right)^{\zeta}$ $(\alpha, \beta, \xi, \zeta-$ постоянные величины). В результате имеем следующую эмпирическую формулу для расчета значения прецессии перицентра:

$$
\dot{\omega}_{e}=10.7(1) \times \frac{G^{2 / 3}\left(M_{1}+M_{2}\right)^{2 / 3}}{w^{2} P_{b}^{5 / 3}\left(1-e^{2}\right)} .
$$

Принимая во внимание, что $(2 \pi)^{5 / 3} / 2=10.697, w=c / \sqrt{6}$ имеем:

$$
\dot{\omega}_{e} \approx \frac{3 G^{2 / 3}\left(M_{1}+M_{2}\right)^{2 / 3}}{c^{2}\left(\frac{P_{b}}{2 \pi}\right)^{5 / 3}\left(1-e^{2}\right)} .
$$

Правая часть формулы (11) полностью совпадает с формулой для прецессии перицентра в общей теории относительности [4] и (в случае $M_{1} \gg M_{2}$ или $\left.M_{1} \ll M_{2}\right)-$ в теории Гербера [24].

\section{III. ПРЕЦЕССИЯ ЦЕНТРОВ МАСС ДВОЙНЫХ СИСТЕМ}

Сила, действующая со стороны 1-го компонента на 2-ой, не равна силе, действующей со стороны 2-го компонента на 1-ый. В результате на центр масс системы действует сила $F_{c}$.

Для дальнейшего анализа необходимо определиться с системой отсчета и задаться направлениями движений компонентов по своим орбитам. За начальное примем положение, при котором компоненты находятся в апоцентрах своих орбит, компонент с массой $M_{1}$ находится справа от центра масс системы, компонент с массой $M_{2}$ - слева; компоненты движутся по своим орбитам против часовой стрелки; ось $x$ направлена слева направо, ось $y$ - снизу вверх; отсчет угла $\theta$ между осью $x$ и радиус-вектором 1-го компонента ведется от положительного направления оси $x$.

Определим силу $F_{c}$ :

$$
F_{c}=F_{2 \leftarrow 1}-F_{1 \leftarrow 2}=\frac{G M_{1} M_{2}}{r^{2}} \frac{\kappa\left(v_{1}^{2}-v_{2}^{2}\right)}{w^{2}},
$$


где $r=a\left(1-e^{2}\right)(1-e \cos \theta)^{-1}(e-$ эксцентриситет системы, $a-$ большая полуось относительной орбиты системы),

$$
\begin{aligned}
& v_{1}=\frac{1}{\sqrt{1-e^{2}}} \frac{M_{2}}{M_{1}+M_{2}} \sqrt{\frac{G\left(M_{1}+M_{2}\right)}{a}} \sqrt{1+e^{2}-2 e \cos \theta}, \\
& v_{2}=\frac{1}{\sqrt{1-e^{2}}} \frac{M_{1}}{M_{1}+M_{2}} \sqrt{\frac{G\left(M_{1}+M_{2}\right)}{a}} \sqrt{1+e^{2}-2 e \cos \theta} .
\end{aligned}
$$

После подстановки получаем:

$$
F_{c}=\frac{G^{2} M_{1} M_{2}\left(M_{1}-M_{2}\right) \kappa}{a^{3} w^{2}\left(1-e^{2}\right)^{3}}(1-e \cos \theta)^{2}\left(1+e^{2}-2 e \cos \theta\right) .
$$

Найдем среднее за период $P_{b}$ системы значение $F_{c}$ (в предположении отсутствия прецессии перицентра):

$$
\overline{F_{c}}=\frac{1}{P_{b}} \int_{0}^{P_{b}} F_{c} d t=\frac{1}{P_{b}} \int_{0}^{L} F_{c} \frac{d l}{v}
$$

где $L-$ периметр относительной эллиптической орбиты, $d l-$ элементарный путь по относительной орбите,

$$
\begin{gathered}
v=v_{1}+v_{2}=\frac{1}{\sqrt{1-e^{2}}} \sqrt{\frac{G\left(M_{1}+M_{2}\right)}{a}} \sqrt{1+e^{2}-2 e \cos \theta} \\
d l=a\left(1-e^{2}\right) \frac{\sqrt{1+e^{2}-2 e \cos \theta}}{(1-e \cos \theta)^{2}} d \theta ; \quad a=\sqrt[3]{\frac{G P_{b}^{2}\left(M_{1}+M_{2}\right)}{4 \pi^{2}}} . \\
\frac{d l}{v}=\frac{P_{b}}{2 \pi} \frac{\left(1-e^{2}\right)^{3 / 2}}{(1-e \cos \theta)^{2}} d \theta .
\end{gathered}
$$

Среднее (по времени) значение проекции силы $F_{c}$ на ось $x$ (в предположении отсутствия прецессии перицентра):

$$
\begin{gathered}
\overline{F_{c X}}=\frac{2 \pi \kappa}{\left(1-e^{2}\right)^{3 / 2}} \frac{G M_{1} M_{2}}{P_{b}^{2} w^{2}} \frac{M_{1}-M_{2}}{M_{1}+M_{2}} \int_{0}^{2 \pi} \cos \theta\left(1+e^{2}-2 e \cos \theta\right) d \theta= \\
=\frac{4 \pi^{2} \kappa e}{\left(1-e^{2}\right)^{3 / 2}} \frac{G M_{1} M_{2}}{P_{b}^{2} w^{2}} \frac{M_{2}-M_{1}}{M_{1}+M_{2}} .
\end{gathered}
$$

Среднее (по времени) значение проекции силы $F_{c}$ на ось $y$ (в предположении отсутствия прецессии перицентра):

$$
\overline{F_{c Y}}=\frac{2 \pi \kappa}{\left(1-e^{2}\right)^{3 / 2}} \frac{G M_{1} M_{2}}{P_{b}^{2} w^{2}} \frac{M_{1}-M_{2}}{M_{1}+M_{2}} \int_{0}^{2 \pi} \sin \theta\left(1+e^{2}-2 e \cos \theta\right) d \theta=0 .
$$


Таблица V. Параметры циклоидальных траекторий движения центров масс двойных систем, $\times 10^{12}$ м

\begin{tabular}{c|c|c||c|c|c}
\hline PSR & высота & шаг & PSR & высота & шаг \\
\hline $\mathrm{B} 1913+16$ & 11.24 & 35.36 & J0737-3039 & 0.797 & 2.51 \\
$\mathrm{~B} 2127+11 \mathrm{C}$ & 0.952 & 3.00 & J1756-2251 & 9.48 & 29.82 \\
$\mathrm{~J} 1906+0746$ & 0.516 & 1.62 & J1141-6545 & 12.49 & 39.29 \\
\hline
\end{tabular}

В результате имеем:

$$
\overline{F_{c}}=\overline{F_{c X}}=\frac{4 \pi^{2} \kappa e}{\left(1-e^{2}\right)^{3 / 2}} \frac{G M_{1} M_{2}}{P_{b}^{2} w^{2}} \frac{M_{2}-M_{1}}{M_{1}+M_{2}} .
$$

Сила $F_{c}$, действующая на центр масс системы, направлена от перицентра орбиты тяжелого компонента к перицентру орбиты легкого компонента. Прецессия перицентров орбит приведет к повороту силы $F_{c}$ по направлению вращения компонентов (т.е. в выбранной системе отсчета - против часовой стрелки). В результате центр масс двойной системы будет двигаться по циклоидальной траектории с периодически изменяющейся скоростью. Уравнение движения центра масс в параметрическом виде (принимаем скорость прецессии $\dot{\omega}$ постоянной):

$$
\left\{\begin{array}{l}
x(t)=\frac{h}{\dot{\omega}^{2}}(1-\cos (\dot{\omega} t)) \\
y(t)=\frac{h}{\dot{\omega}^{2}}(\dot{\omega} t-\sin (\dot{\omega} t))
\end{array}\right.
$$

где

$$
h=\frac{4 \pi^{2} \kappa e}{\left(1-e^{2}\right)^{3 / 2}} \frac{G M_{1} M_{2}}{P_{b}^{2} w^{2}} \frac{M_{2}-M_{1}}{\left(M_{1}+M_{2}\right)^{2}} .
$$

На рис. 1 показаны траектории движения центров масс некоторых двойных систем.

Как можно видеть, они представляет собой циклоиды. Данные по высоте и шагу этих циклоид приведены в табл. V.

Скорость $v_{c}$, максимальная скорость $v_{c}^{\max }$ и средняя (по времени) скорость $\overline{v_{c}}$ движения центра масс определятся следующим образом:

$$
v_{c}=\frac{\sqrt{2} h}{\dot{\omega}} \sqrt{1-\cos (\dot{\omega} t)} ; v_{c}^{\max }=\frac{2 h}{\dot{\omega}} ; \overline{v_{c}}=\frac{4 h}{\pi \dot{\omega}} .
$$

Обнаружение периодического изменения скорости движения центра масс системы явится дополнительным подтверждением описываемой в данной статье зависимости гравитации от скорости источника и «приемника». 
$\mathrm{B} 1913+16$

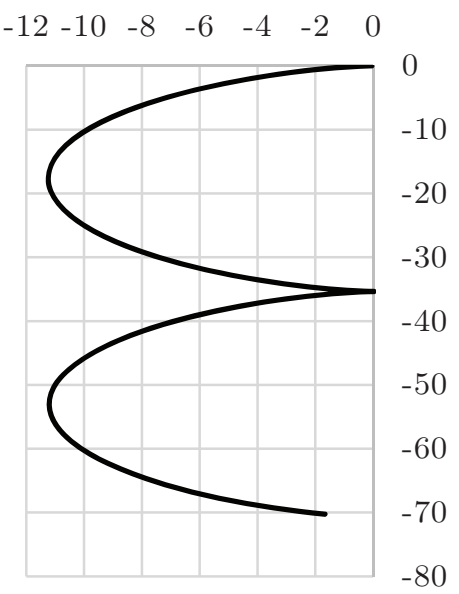

$$
\mathrm{J} 1906+0746
$$

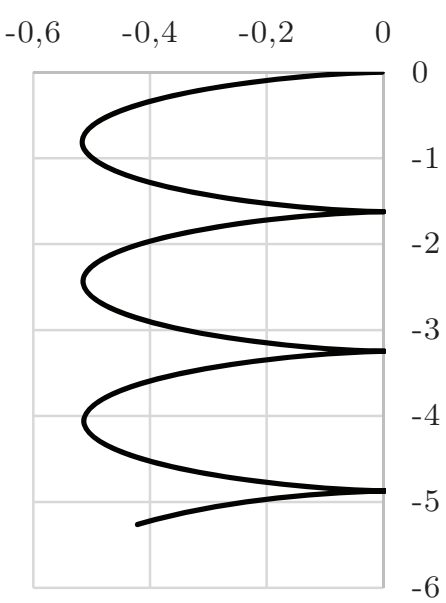

J0737-3039

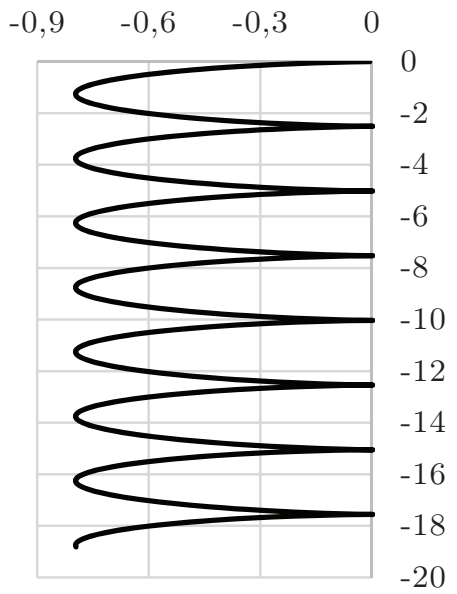

$\mathrm{J} 1141+6545$

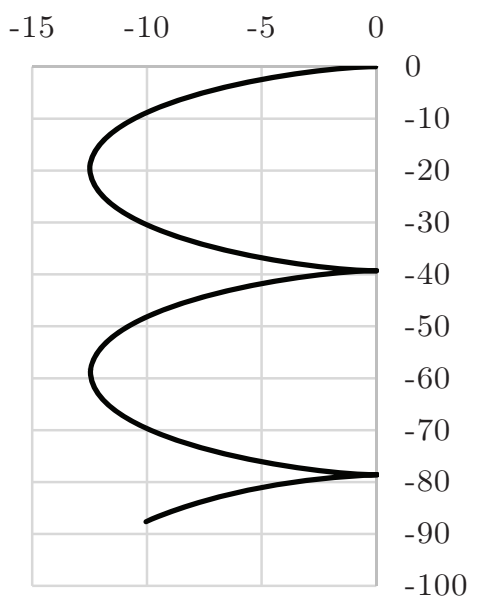

J1756-2251

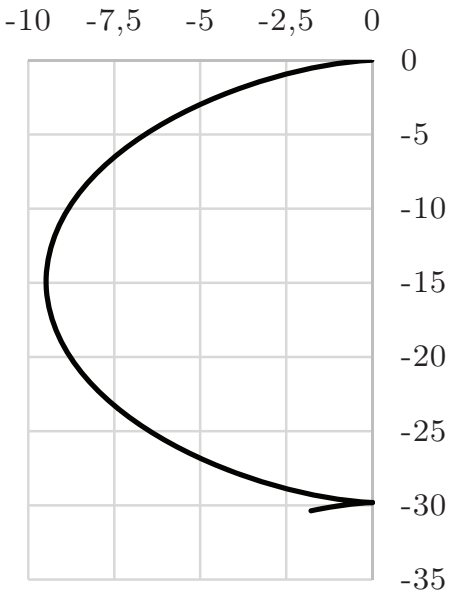

$\mathrm{B} 2127+11 \mathrm{C}$

$-1 \quad-0,75 \quad-0,5 \quad-0,25 \quad 0$

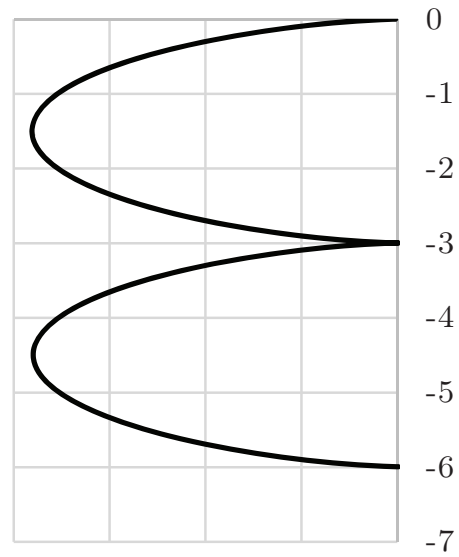

Рис. 1. Траектории движения центров масс двойных систем за 160 лет, $\times 10^{12}$ м

Наименьшим периодом изменения скорости движения центра масс (из числа систем, по которым имеются достоверные данные по массам компонентов, табл. VI) обладает система PSR J0737-3039. Период $P_{c}$ изменения скорости центра масс этой системы равен 21.3 года, максимальное значение скорости $-1.854 \mathrm{~km} /$ сек, среднее значение скорости $-1.18 \mathrm{kM} /$ сек.

Точность измерения скорости движений центров масс двойных систем в настоящее время невысокая. Согласно [25] скорость движения центра масс системы PSR J07373039 в плоскости системы составляет $96.0 \pm 3.7 \mathrm{kM} / \mathrm{c}$. Погрешность определения этой скорости, как можно видеть, в 2 раз больше максимальной скорости периодического движения центра масс. 
Таблица VI. Прецессия центров масс некоторых двойных систем

\begin{tabular}{l|c|c|c}
\hline PSR | Параметр & $P_{c}=2 \pi / \dot{\omega}$, лет & $v_{c}^{\max }, \mathrm{kм} /$ сек & $\overline{v_{c}}, \mathrm{kм} /$ сек \\
\hline B1913+16 & 85.2 & 6.553 & 4.172 \\
J0737-3039 & 21.3 & 1.854 & 1.180 \\
B2127+11C & 80.6 & 0.323 & 0.206 \\
J1756-2251 & 139.4 & 3.370 & 2.146 \\
J1906+0746 & 47.5 & 0.539 & 0.343 \\
J1141-6545 & 67.8 & 9.053 & 5.763 \\
\hline
\end{tabular}

Движение центра масс двойных систем может быть выявлено из наблюдения за долгопериодическими колебаниями лучевой скорости тяжелого компонента двойной системы (т.е. наблюдаемой скорости приближения или удаления тяжелого компонента от наблюдателя за вычетом постоянной составляющей, связанной с инерционным движением центра масс системы). Выбор тяжелого компонента обусловлен тем, что его скорость меньше, поэтому амплитуда относительных колебаний скорости выше.

Для определения текущей скорости тяжелого компонента определим значения проекций на оси $x$ и $y$ скорости орбитального движения $\left(v_{1 x}, v_{1 y}\right)$ и переменной скорости движения центра масс $\left(v_{c x}, v_{c y}\right)$.

Определим вначале проекции орбитальной скорости тяжелого компонента:

$$
\begin{gathered}
v_{1 x}=v \cos (u-\theta)=v(\cos u \cos \theta+\sin u \sin \theta)=v_{0} \sin \theta ; \\
v_{1 y}=v \sin (u-\theta)=v(\sin u \cos \theta-\cos u \sin \theta)=v_{0}(\cos \theta-e),
\end{gathered}
$$

где $\theta$ - угол между радиус-вектором тяжелого компонента и осью $x ; u$ - угол между радиус-вектором тяжелого компонента и орбитальной скоростью:

$$
\begin{gathered}
\cos u=\frac{e \sin \theta}{\sqrt{1+e^{2}-2 e \cos \theta}}, \sin u=\frac{1-e \cos \theta}{\sqrt{1+e^{2}-2 e \cos \theta}} ; \\
v_{0}=\frac{1}{\sqrt{1-e^{2}}} \frac{M_{2}}{M_{1}+M_{2}} \sqrt{\frac{G\left(M_{1}+M_{2}\right)}{a}}
\end{gathered}
$$

где $M_{2}$ - масса легкого компонента.

Значения проекций переменной скорости центра масс:

$$
v_{c x}=H \sin \varphi ; v_{c y}=H(1-\cos \varphi),
$$

где $H=h / \dot{\omega}$. 

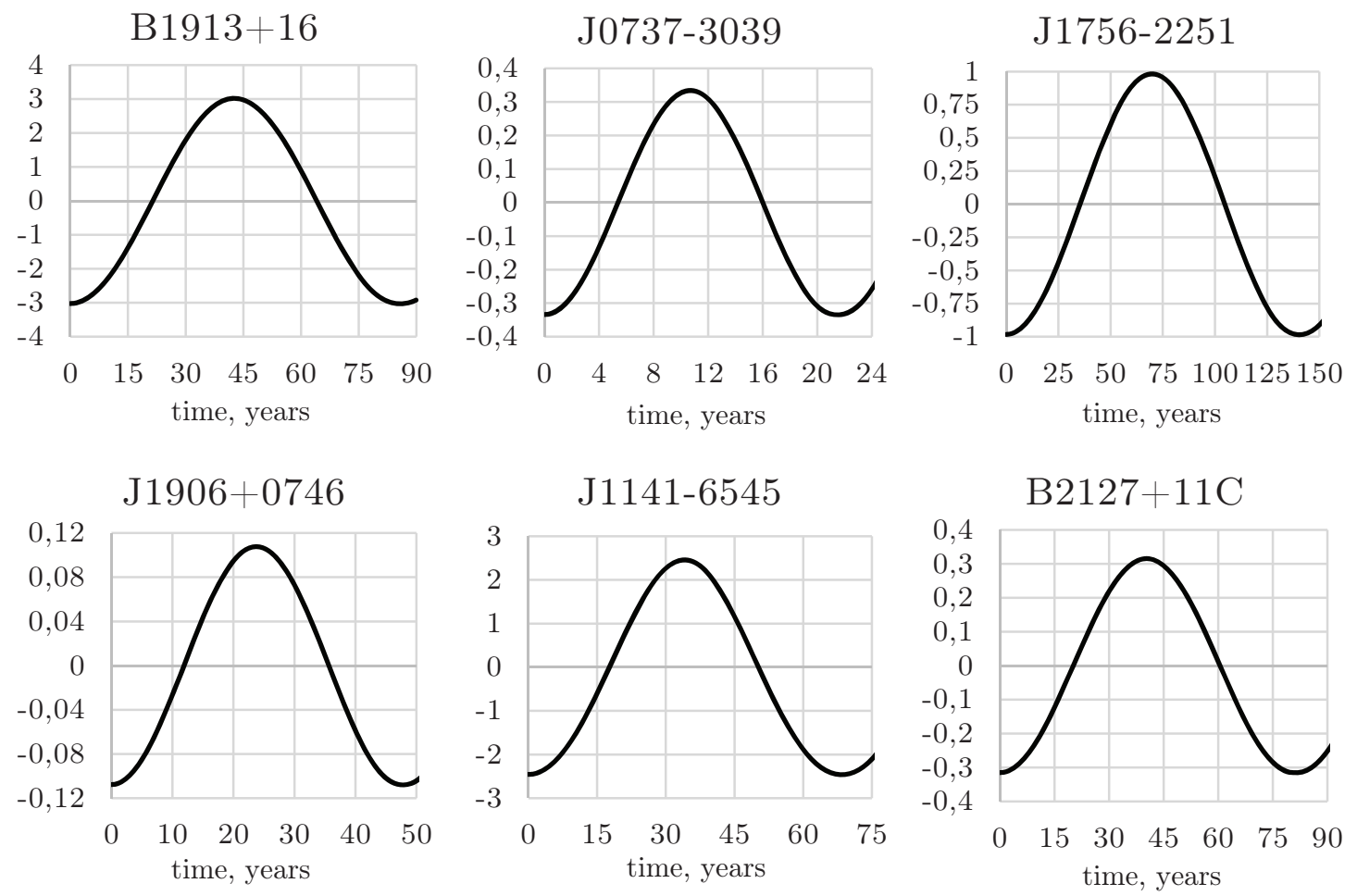

Рис. 2. Долгопериодические колебания минимальной лучевой скорости тяжелого компонента, $\%$

Суммарная скорость тяжелого компонента двойной системы:

$$
v=\sqrt{\left(v_{0} \sin \theta+H \sin \varphi\right)^{2}+\left(v_{0}(\cos \theta-e)+H(1-\cos \varphi)\right)^{2}} .
$$

Определим значения угла $\theta_{0}$, при котором (при заданном значении $\varphi$ ) функция скорости $v$ имеет экстремумы:

$$
\frac{d v}{d \theta}=0 \Rightarrow \theta_{0}=-\arctan \left(\frac{H \sin \varphi}{H \cos \varphi-v_{0} e-H}\right) .
$$

Определим теперь значения угла $\varphi_{0}$, при котором (при $\left.\theta=\theta_{0}\right)$ функция скорости $v$ имеет экстремумы:

$$
\frac{d v}{d \varphi}=0 \Rightarrow \varphi_{0}=0, \pi
$$

В результате имеем:

$$
v_{\max }(\varphi=\pi)=v_{0}(1-e)+2 H ; v_{\min }(\varphi=0)=v_{0}(1-e) .
$$


Относительная амплитуда колебаний минимальных значений скорости тяжелого компонента (равная амплитуде относительных колебаний его лучевой скорости):

$$
\Delta v= \pm \frac{v_{\max }-v_{\min }}{v_{\max }+v_{\min }}= \pm \frac{H}{v_{0}(1-e)+H} .
$$

Расчет по формуле (26) дает следующие значения:

\begin{tabular}{c|c||c|c||c|c}
\hline PSR & $\Delta v, \%$ & PSR & $\Delta v, \%$ & PSR & $\Delta v, \%$ \\
\hline B1913+16 & \pm 3.03 & B2127+11C & \pm 0.31 & J1906+0746 & \pm 0.11 \\
J0737-3039 & \pm 0.33 & J1756-2251 & \pm 0.98 & J1141-6545 & \pm 2.45 \\
\hline
\end{tabular}

Полученные значения подтверждаются результатами моделирования (см. рис. 2).

\section{IV. ЗАКЛЮЧЕНИЕ}

Совпадения с действительностью значений прецессии перицентров орбит, полученных в результате численного моделирования, является весомым аргументом в пользу предлагаемой зависимости гравитации от скорости взаимодействующих тел (см. (9)). Указанное совпадение результатов моделирования с действительностью наблюдается во всех двойных системах, в которых не проявляются в существенной степени прочие факторы, влияющие на прецессию перицентра орбиты (потеря массы, приливные эффекты и т.д.).

Согласно подтвержденной в данной статье зависимости (9) при гравитационном взаимодействии тел в общем случае сила гравитационного воздействия на 2-ое тело со стороны 1-го не равна силе гравитационного воздействия на 1-ое тело со стороны 2го. Принимая во внимание третий закон Ньютона указанное различие сил возможно только в случае, когда гравитационное взаимодействие осуществляется не напрямую между телами, а через какого-либо посредника, например среду («физический вакуум», «эфир» и т.п.). В этом случае соблюдение третьего закона Ньютона обеспечивается во взаимодействиях 1-го тела со средой и 2-го тела со средой.

Для окончательного подтверждения определенной в данной статье зависимости силы гравитационного взаимодействия от скорости движения необходимо практическое обнаружение движений центров масс двойных систем по рассчитанным циклоидальным траекториям. В настоящее время такого подтверждения пока не имеется. По мере накопления результатов измерений лучевых скоростей двойных систем возможности обнаружить ожидаемый эффект будут неуклонно повышаться. 


\section{СПИСОК ЛИТЕРАТУРЫ}

[1] Вейсберг и др. (J. M. Weisberg et al.) ASP Conf.Ser. 328, 25 (2005) arXiv:astro-ph/0407149v1

[2] Бургей и др. (M. Burgay et al.) Nature 426, 531 (2003) arXiv:astro-ph/0312071v1

[3] Стэйрс и др. (I. H. Stairs et al.) ApJ 581, 501 (2002) arXiv:astro-ph/0208357v1

[4] Джекоби и др. (В. А. Jacoby et al.) ApJ 644, L113 (2006) arXiv:astro-ph/0605375v1

[5] Янсен и др. (G. H. Janssen et al.) A\&A 490, 753-761 (2008) arXiv:astro-ph/0808.2292v1

[6] Миньяни и др. (R. P. Mignani et al.) Mon.Not.Roy.Astron.Soc. 430 (2), 1008 (2013) arXiv:astro-ph/1212.4801v1

[7] Лайн и др. (A.G. Lyne et al.) Mon.Not.Roy.Astron.Soc. 312, 698 (2000) arXiv:astro-ph/9911313v1

[8] Коранджу и др. (A. Corongiu et al.) A\&A 462, 703 (2007) arXiv:astro-ph/0611436v1

[9] Мартинес и др. (J. G. Martinez et al.) ApJ 812, 143 (2015) arXiv:astro-ph/1509.08805v1

[10] Фердман и др. (R. D. Ferdman et al.) Mon.Not.Roy.Astron.Soc. 443 no.3, 2183 (2014) arXiv:astro-ph/1406.5507v1

[11] Лиувен и др. (J. van Leeuwen et al.) ApJ 798, 118 (2015) arXiv:astro-ph/1411.1518v1

[12] Чемпион и др. (D. J. Champion et al.) Mon.Not.Roy.Astron.Soc. 350, L61 (2004) arXiv:astro-ph/0403553v1

[13] Чемпион и др. (D. J. Champion et al.) Mon.Not.Roy.Astron.Soc. 363, 929 (2005) arXiv:astro-ph/0508320v2

[14] Свиггам и др. (J. K. Swiggum et al.) ApJ 805, 156 (2015) arXiv:astro-ph/1503.06276v1

[15] Вёрбиест и др. (J. P. W. Verbiest et al.) The Twelfth Marcel Grossmann Meeting (Thibault Damour, Robert Jantzen and Remo Ruffini. Paris, World Scientific, 2012, 1571) arXiv:astro-ph/1210.0224v1

[16] Бейлис и др. (M. Bailes et al.) АpJ 595 L49 (2003) iopscience.iop.org/article/10.1086/378939/pdf

[17] Дэвис и др. (M. B. Davies et al.) Mon.Not.Roy.Astron.Soc. 335(2), 369 (2002) arXiv:astro-ph/0204511v1

[18] Тушет и др. (S. E. Thorsett et al.) ApJ 405, L29 (1993) arXiv:astro-ph/9303002v2

[19] Тушет и др. (S. E. Thorsett et al.) ApJ 512, 288 (1999) iopscience.iop.org/article/10.1086/306742/pdf

[20] Сплавер и др. (Е. M. Splaver et al.) ApJ 581, 509 (2002) arXiv:astro-ph/0208281v1

[21] Фреири и др. (Р. С. С. Freire et al.) Mon.Not.Roy.Astron.Soc. 412, 2763 (2011) arXiv:astro-ph/1011.5809v1 
[22] Каргхария и др. (J. Khargharia et al.) ApJ 744, 183 (2012) arXiv:astro-ph/1110.0507v1

[23] Кевин Браун (Kevin Brown) Reflections on Relativity (Lulu Enterprises Incorporated, 2016. p. 416)

[24] П. Гербер (Paul Gerber) Zeitschrift für Mathematik und Physik. 43, 93 (1898) archive.org/details/zeitschriftfrma14runggoog/page/n101/mode/2up

[25] Рэнсом и др. (S. M. Ransom et al.) ApJ 609, L71-L74 (2004) arXiv:astro-ph/0404149v1 